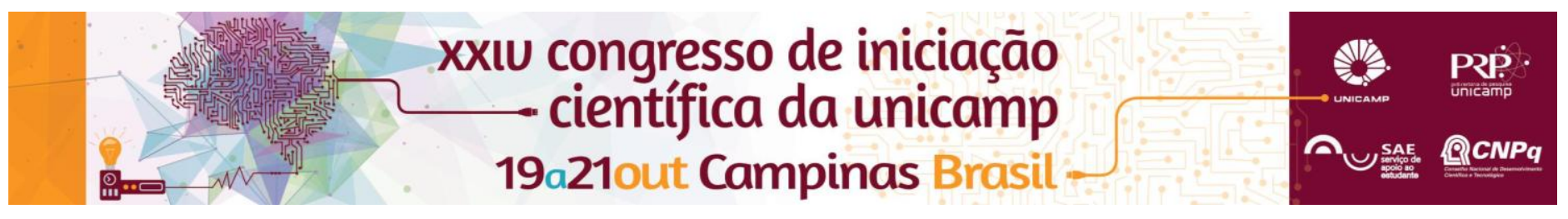

\title{
Controlled Drug Release by Amphiphilic Polyurethane Hydrogels
}

\author{
Lucas Polo da Fonseca*, Rafael Bergamo Trinca, Maria Isabel Felisberti.
}

\begin{abstract}
Amphiphilic polyurethanes (PU), composed by PEG as hydrophilic segment and PCL-triol as hydrophobic crosslinking agent, were loaded with sodium diclofenac, a anti-inflammatory drug, using ethanol solutions. The drug release in PBS buffer solution was investigated. The sodium diclofenac sorption in the hydrogels increased with the drug concentration in the loading solutions and decreased with increasing crosslinking density of the hydrogels. The drug release in PBS solutions achieved a linear dependence with time after 50 minutes of water swelling, indicating a controlled release behaviour.
\end{abstract}

\section{Key words: \\ hydrogels, controlled drug release, polyurethanes.}

\section{Introduction}

Polyurethane hydrogels are materials of great interest in the biomedical field due to their potential biocompatibility, biodegradability, and appropriate mechanical properties ${ }^{1}$. There are studies about drug loading and release from polyurethane hydrogels ${ }^{2}$. Fonseca and coworkes ${ }^{3}$ reported the synthesis and characterization of amphiphilic polyurethanes composed by poly(ethylene glicol) (PEG), polycaprolactone diol (PCL-diol) and polycaprolactone triol (PCL-triol) as crosslinking agent. These polyurethanes presented high water swelling coefficient, becoming hydrogels that presented high resilience, thermo responsiviness and a potential for controlled drug release, as further demonstrated herein.

\section{Results and Discussion}

Drug loading tests were conducted with the PU using ethanol solutions of sodium diclofenac, a hydrophobic nonsteroidal anti-inflammatory drug contraindicated to patients that have stomach illness. Sodium diclofenac uptake, express as the mass fraction of drug in the dried polyurethane $\left.\left[\mathrm{m}_{\mathrm{drug}} / \mathrm{m}_{\mathrm{PU}}\right) \%\right]$, increased linearly with increasing drug concentration in ethanol solutions, Figure 1(a). However, sodium diclofenac uptake was more pronnounced for polyurethane containing 89 wt\% of PEG and 11 wt\% of PCL-triol possibily due to its lower crosslinking density and consequently higher diclofenac solution uptake. Moreover, the addition of 5 wt\% of diclofenac to ethanol increased the swelling coefficient compared to neat ethanol, and further drug addition did not influence the swelling of PU. A remarkable result is referent to the drug partition. After reaching swelling equilibrium, the concentration of drug in the swollen PU is higher than in the ethanol solution. This result is expressed by the partition coefficient presented in Figure 1(a). PU containing the drug were subject to water swelling experiments, becoming hydrogels, and drug release studies, monitored by in situ UV spectroscopy. Results are shown in Figure 1(b). Water swelling equilibrium was achieved after 250 min for both PU. Drug release in a PBS buffer solution $(\mathrm{pH}=7,4)$, at $25^{\circ} \mathrm{C}$, occurred simultaneosly with water swelling, however, only $5 \%$ of the total drug amount in the hydrogel was released after $250 \mathrm{~min}$. After an initial slow rate in the first $50 \mathrm{~min}$ the drug release followed a linear pattern (Figure 1b), suggesting a controlled release behaviour without burst effect. Release also showed to be composition dependent: PU with $21 \%$ PCL mass ratio presented slower release rate than PU with $11 \%$ of PCL.
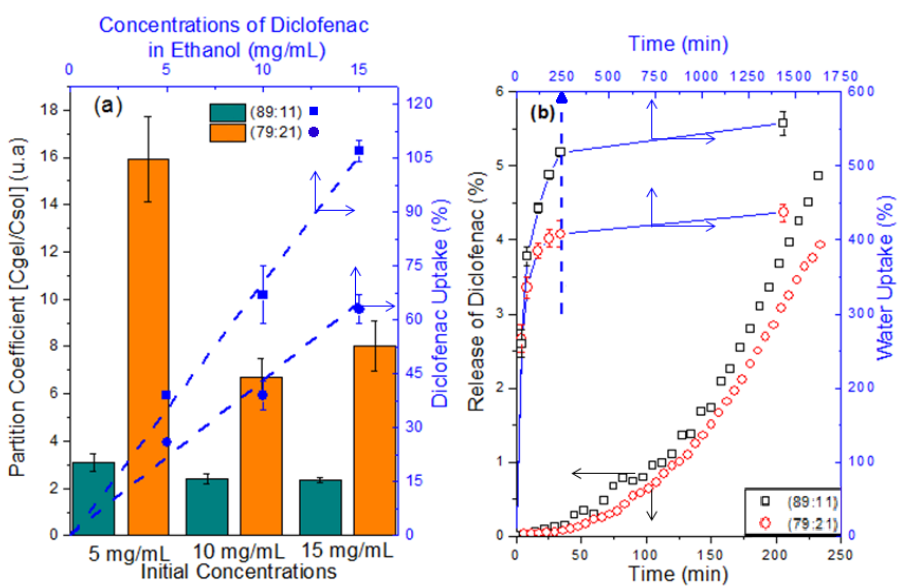

Figure 1. (a) Partition coefficient vs Initial concentrations of drug solutions (columns); Diclofenac Uptake vs Initial concentrations of drug solutions (blue dashed line and dots). (b) Release of Diclofenac vs Time are presented in dots. In blue line and dots is Water uptake vs Time.

${ }^{*}$ Composition of polyurethanes are expressed in terms of mass fraction being: (mPEG(\%):mPCL-triol(\%))

\section{Conclusions}

The PU presented affinity with diclofenac being able to absorb high amount of the drug. The drug release in a PBS buffer solution did not presented a burst effect but an almost constant release rate after 50 min of swelling, suggesting a controlled drug release behavior.

\section{Acknowledgement}

Authors thank the financial support of CNPq-Pibic (Proc. 23255/2015-5) and FAPESP (Proc. 2010/17804-7)

\footnotetext{
${ }^{1}$ Z. Li, Z. Zhang.: Biomacromolecules, 2012, 13, 3977-3989

${ }^{2}$ Paris, R., Fernandes, M. Á., Garrido, Q. I.: Polym. Adv. Techno, 2013, 24, $1062-1067$

${ }^{3}$ Fonseca, L.P., Trinca, R.B., Felisberti, M.I, J. Appl. Polym. Sci., 2016, DOI: 10.1002/APP.43573
} 